\title{
The use of Geocoding for Home Healthcare Application and Management an Epidemic Situation. Case of COVID-19 Virus Outbreak
}

\author{
Ahmed AkakbaA, Belkacem Lahmar ${ }^{\mathrm{A}}$ \\ Received: August 20, 2020 | Revised: October 21, 2020 | Accepted: November 27, 2020 \\ doi: 10.5937/gp24-28062
}

\begin{abstract}
The lack of an addressing system is one of the problems of urban management in Algeria, which makes it hard to find the addresses concerned, especially in case of crisis where the decision-makers need accurate data in real-time. Like many countries, Algeria follows up the world health organization guidelines that declared the COVID-19 virus as pandemic and recommended the full quarantine and reduces the social contact as much as possible; however, these procedures weren't enough to control the increasing number of confirmed cases, which exceeded the hospital's capacities. To face up the outbreak of this pandemic, the Algerian health professionals decided to treat most coronavirus cases at home. This study aims to use a geocoding tool developed in C\# programming language and ArcGIS Software Development Kit (SDK) to help in the epidemiological control operation in Ain Touta city and simplifies the interventions using a spatial approach. These problems are addressed by a tool to collect, analyze, store, and process archiving of the geographic data using a geodatabase server.
\end{abstract}

Keywords: Geocoding; COVID-19; quarantine; address; home healthcare

\section{Introduction}

The Algerian cities are mosaics with random and uncontrolled urban evolution for many reasons (economics, politics, security, historic...etc.), so the territory control and data flow such as the addresses is difficult to handle due lack of accurate information, the local government of many cities finds problems to implement coherent management, weaknesses of the territory control, and the lack of coordination (Akakba \& Filali 2017).

By March 2020, Algeria was detected the first confirmed COVID-19 case, after few days the government announces the province of Blida under full quarantine for $24 / 24$ hours and 7/7 days and till now (by 24th July 2020) over $80 \%$ of the country under the health quarantine. After the outbreak of the COVID-19 vi- rus in Ain Touta and recording more than 200 confirmed cases, the local hospital reaches its maximum capacity (50 beds). This epidemiological situation in Ain Touta forced the decision-makers to choose the home healthcare for stable Coronavirus cases where the control of their health condition is done by sending the medical staff to houses for monitoring the health condition of the patients.

Our paper suggests using a graphical user interface (GUI) tool programmed on the C\# programming language and ArcGIS SDK to use the geocoding technology, and take the advantages of the addresses to simplify the home healthcare, at the same time limiting the spread of the epidemic by applying the social isolation principle. 


\section{Literature review}

The process to convert addresses into geographic locations is known as geocoding, which is a basic operation in geographic information systems (GIS) (Longley et al. 2005), it's widely using in space management (Davis \& Fonseca, 2007; Edwards et al., 2014; Zandbergen, 2014), healthcare and epidemiologic (Krieger et al., 2002; Mazumdar et al., 2008; Oliver et al., 2005), even in civil security (Ratcliffe 2004).

Today, the Internet is affecting GIS in three major areas: access to GIS data is now simpler than before, spatial information dissemination, and GIS modeling/processing. It provides GIS users easy access to GIS data from different data sources and improve data "infrastructure" for providing a basis for valid GIS studies (Yang et al., 2004), because of GIS servers available on the internet such as GeoServer, MapServer, Mapnik, MapGuide, QGIS server, etc.

Online geocoding become a good example of this development and increase its functionality to the Internet users (Roongpiboonsopit \& Karimi 2010). all these servers are open source servers i.e. freely available. ArcGIS also provides server but it is not available for free, but with many extra on features. The most used services are Google maps and OpenStreetMap but there is a difference in their quality (Präger et al., 2019).

Geocoding is available to implement in three addresses models: as a points, parcels, and as a street networks models; but the geocoding quality have been compared each other in many studies such (Zandbergen, 2014) who compares the previous address data models and find that out of 163,886 original samples of addresses, $36 \%$ weren't used in the final analysis because they could not be reliably geocoded using either street or parcel geocoding. Also, according to (Zandbergen, 2007) that used geocoding to detect quality on environmental exposure assessment of children living near high traffic roads (measure pollution on individual address, the result is very accurate) conclude that "Results of the case-study presented here strongly suggest that typical street geocoding is insufficient for fine-scale analysis and more accurate alternatives need to be considered".

There are many potential problems with street geocoding, which have been well described by (Ratcliffe, 2001; Ward et al., 2005; Whitsel et al., 2004; 2006), several studies (Bonner et al., 2003; Cayo \& Talbot, 2003; Dearwent et al., 2001; Karimi et al., 2004) have determined to quantitative estimates of the positional accuracy of street geocoding by comparing the street geocoded locations with the 'true' location based on taking field measurements using a Global Positioning Systems (GPS) unit, The results in urban areas are generally more accurate than in rural areas. This suggests that the positional error of street geocoding can be substantial and needs to be characterized in a meaningful manner relevant to the use of the geocoded locations.

\section{Study area}

The city of Ain Touta is located at the intersection between several important roads, particularly National Road number 03 (RN03), connecting Constantine and Biskra, National Road number 28 (RN28) who linking M'sila and Batna city, as well as the Constantine-Biskra railway line. It's the third most populated city in the province with more than 100000 inhabitants in the city.

Ain Touta is at $450 \mathrm{~km}$ in the South-East of the Algiers capital, between $6^{\circ} 7^{\prime}$ to $6^{\circ} 13^{\prime}$ east longitude and $35^{\circ} 34^{\prime}$ to $35^{\circ} 31$ north latitude, it's located between two mountain ranges with an altitude ranging between 900 and 950 meters.

Ain Touta has one hospital with a capacity of only 50 beds to serve about 100,000 people, as a result, the local health sector of the city suffers from high pressure, which is negatively affecting the quality of the health service, in addition to the spread of the Coronavirus, that threatens the global health system, not to mention the local ones.

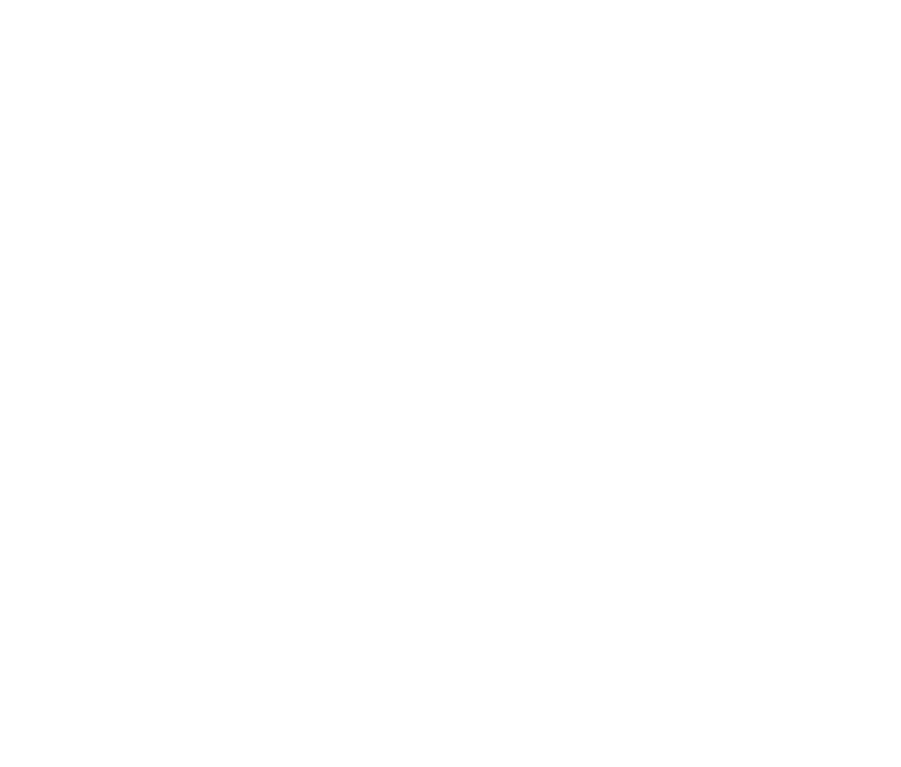

Figure 1. Geographic location of the study area and the user interface of the tool 
The current outbreak caused pressure on local governments, as a result the health infrastructures become unable to take care all coronavirus cases, since the first case recorded in the city on June 1, 2020, raising the number on June 14 to 11 cases, and by the end of the month of June, hundreds of cases recorded. And hundreds of unconfirmed cases.

By 20 June the local hospital reaches its maximum capacity and the local health department proposed the "Home healthcare" for Coronavirus cases, and the medical staff check the patients periodically at home.

To handle the home healthcare management, we propose to create a geographic database to store attributes of every confirmed case and managed by an application that using the geocoding technic.

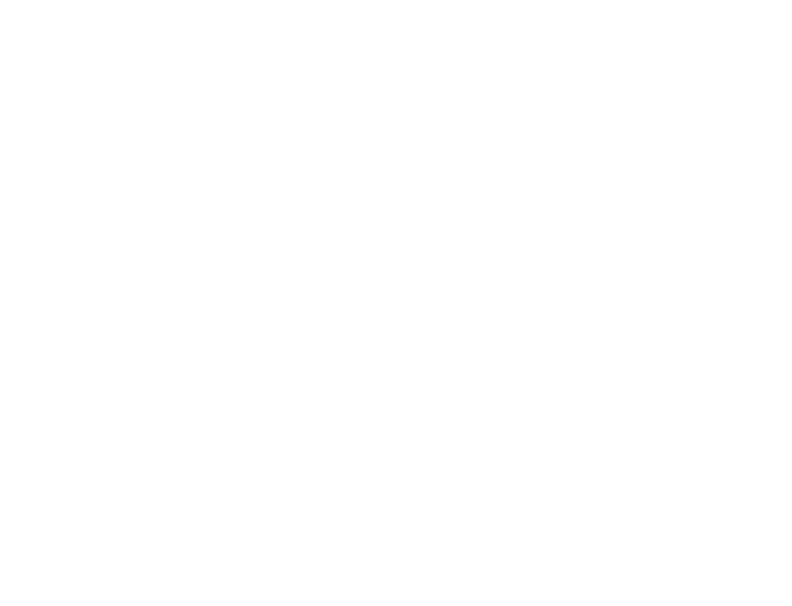

Figure 2. Spread of covid-19 between 1 June - 1 July 2020 in Ain Touta

\section{Address data modelling}

There are many challenges to building a good reference data (Arctur \& Zeiler, 2004; Yang et al., 2004), and technically the addresses can be associated with many kinds of feature classes in a reference database; for example, road centerlines, parcel boundaries, address points, building structures (Zandbergen, 2007). We can organize geocoding model into four different models as follow: the geographic model, street network model, parcel data model, and address point model

The geographic unit model can consist of postal codes (such as ZIP codes in the United States), counties, cities, census enumeration areas, or any other geographic boundary considered meaningful. In the geocoding process, the location assigned to a particular address is the polygon (or the polygon centroid) representing the geographic unit. Location within the unit is not specified, but analyses can be carried out using data associated with the geographic unit. In the case of our study area the geographic unit address is refereeing to the administrative boundary and to the neighborhoods (represented by a polygon).

The street network model is most widely employed address data model and based on street network data. In this approach, a street network is represented as street line segments that hold street names and the range of house numbers and block numbers on each side of the street. Address geocoding is accomplished by first matching the street name, then the segment that contains the house numbers, and finally placing a point along the segment based on a linear interpolation within the range of house numbers.

The street network address model facilitates storing different names and address ranges for different sides of the street and enables validation of cases where there is no address range for one side of the street. It also supports cases where streets have multiple address ranges and names. Some additional attribute characteristics include the use of full block address ranges for major roads. While this results in a better spatial location for known valid addresses, this can also be problematic. When approximated addresses are geocoded against the centerline the records fail to match since the value does not fit into the existing range. The street address in Ain Touta is collected from OpenStreetMap server, local government department and from field.

Parcel boundaries is another address model which is traditionally the most spatially accurate data with address information available. Geocoding against parcels allows for matching against individual plots of land (or the centroids of those polygons) rather than interpolating against a street centerline. This is particularly useful in areas where parcels are not regularly addressed (such as on roads with mixed parity) or those parcels that may be quite a distance from the centerline. The parcel boundaries in our study area are not accurate due historical and planning reasons (The parcels are mostly out of control of the local government and was located far from the city at first time so they were not integrated into the official plan of the city and didn't benefit from an official addressing system till now, of course there is a new geocoding system proposed by the Algerian government to solve this situation started earlier this year 2020).

To overcome the limitations of parcels for geocoding, address points have emerged as the main address data model. The address point data model can also be constructed from several existing data layers such as parcel data. The point address model is the most accurate model because it can be constructed from several existing address data and merged into one geocoding 
database, this is called the composite address. This is the preferred model to solve no-structuring address in our study, which is constructed from:

- the geographic address model that represent the administrative and neighborhoods boundaries,

- the street model that store the house number in both side if any,

- partially, the parcel model as this address model is new and not full adopted officially;

\section{Geocoding in Algeria}

Geocoding is the ability to transform the indirect spatial reference of a piece of information into a direct spatial reference, many geocoding web services have been developed to fulfill this need (Google Geocoding API, Mapzen1), public agencies or from the opensource community (OSM Nominatim3, Gisgraphy4). Geocoding in Alegria is classed on the 3rd level on the quality of the address which is the lightest-shaded countries, this level provides a fair geocoding experience. Address searches sometimes result in matches to point address and street address levels, but more often match to the street name and admin levels ("Geocode coverage-ArcGIS REST API: World Geocoding Service | ArcGIS for Developers", 2020).

To understand the address in Algeria and improve its quality, it is essential to answer the following questions:
- What are the obstacles preventing the use of Geocoding in Algeria?

- What type of model should it adopt in Algeria?

- What is the preferred model to standardize addresses in Algeria?

To answer these questions, the approach used consists of analyzing the main models of address geocoding and the possibility of composing a standard model suitable for our cities. This data contains indirect textual references about location, such as place names (toponyms) or postal addresses. To map such data, each item needs to be geocoded, i.e. assigned with coordinates through the matching of an indirect spatial reference with entities identified in a geographical data source (e.g. a map georeferenced in a well-known coordinate reference system) (Goldberg et al., 2007).

This paper aims to develop a geocoding tool to:

- Use ArcGIS SDK. Net framework to handle geocoding information.

- Create a graphic user interface (GUI) to add, edit, update, and find the address.

- A qualification of geocoding results in textual and spatial terms.

- Synchronize database between online server and offline user.

- Solve no-structuring address data and simplify manage the urban space.

\section{Approaches}

This approach is to simplify the management of the addresses geographically as follow:

- Use ArcGIS SDK and C\# programming language to create a desktop user interface (UI) and assure the connection between the UI and server database.

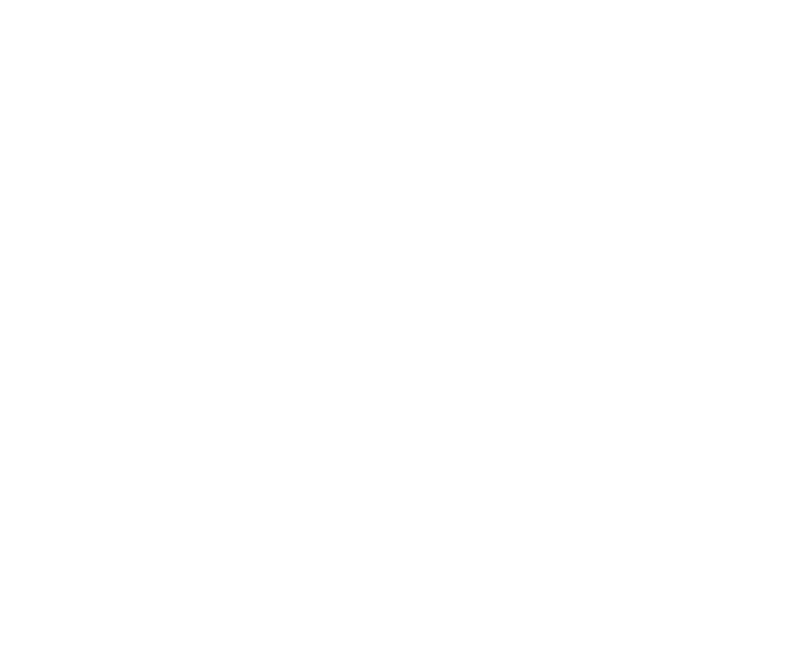

Figure 3. Different parts of the User Interfaces (UI) Tool
- Create Geo-server database: provided the requested data such as base maps.

- The desktop user interface has 4 mains parts:

a) Geographical map that display the map and locations of addresses.

b) Part to display related information current location, current address selected.

c) A part that can add, modify addresses.

d) Assure synchronize data between offline-online geodatabase.

- GeoServer: it hosts all addresses information and locations for every single address, it's stocked online to make it possible to work online or archiving, or save a copy of it offline file (.gpkg). The following figure illustrates the approach taken.

The data is directly associated with geographical coordinates with inputs, output, and algorithms as below:

- Inputs: are the addresses text information such as:12 Mustakbal street, stand, Ain Touta, Algeria) or incomplete information such (al-moujahidine, 
the full name) or (rue louchen taher.) or simply refer to a landmark (theatre de Batna, radio ourass, rahba...etc).

- Outputs: are a referenced geographically dataset that select the location they are most often rendered into simple two-dimensional points associated with inputs.

- The processing algorithm: consists in finding the best-matching element from the reference dataset.

\section{Collecting Data}

The data provided in this this paper are collected from different sources and it's a personnel field survey that started from $01^{\text {st }}$ June 2020 until $1^{\text {st }}$ of July 2020 . The exact Coronavirus confirmed number in the city and the most important the location (the addresses) of patient are officially unavailable because of the patient privacy policy.

It should be mentioned that the personal data was hidden to protect the privacy of the infected patients, but in a way that does not negatively affect the scientific value of this scientific paper.

\section{Locator address}

The locator address is generated previously using GIS software; it's a composite geocoding that merges:

- District locator address: this locator contained the district number of neighborhoods.

- Neighborhood name locator; this is simple locator created for demonstrate purpose, it contains one neighborhood, but we can include all neighborhoods in Ain Touta city.

- Bloc number: a locator represents the bloc number of every bloc.

- The city locator is to represent the city, but as mentioned before in literature review, we can use any

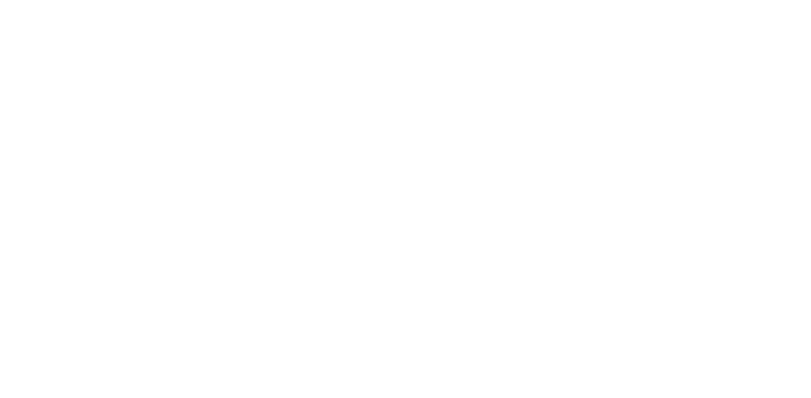

Figure 4. Model and approach

online geocoding server to get the geographic address (state, zip, city...etc.).

The feature to represent the address geographically is the point, according to previous cited studies, the point is the most accurate data and give us very accurate result compared to street and parcel and geographic address.

\section{Open source file}

It's possible to use traditional database file extension ( ${ }^{*}$.shp, geodatabase) in the SDK, but we prefer to convert all files to an (*. gpkg) file. Because Geopackage allow the following features:

- Open source extension.

- Compatible with OGC standard.

- Broadly implemented (GDAL, QGIS, R, Python, Esri, ...)

- More lightweight than a real geodatabase, but as fast as a geodatabase

- Everything is contained in a single file and easier file management with GeoPackage than with Esri Shapefile

- Faster work-flow than with Shapefiles.

- There are nearly no limitations

\section{Resultants and discussions}

The proposed tool has geographical part and descriptive part as we explain before in the approach.

The source code and functions using is explained as follow:

\section{Initiate all necessary files}

In this initiate part (See 'Initiate database and necessary files' section in annex), it uploads all the necessary files and databases, framework...etc. including:

- Address Geo-package file with $\left({ }^{\star}\right.$.gpkg) extension it's a free open source file that hold all attribute table.
- The geocoding $\left({ }^{*}\right.$. loc) file: a file store offline geocoding data in case of unavailable of an online geocoding service.

- Esri Basemaps and online resources,

\section{Add new address and save it as (*.gpkg) file.}

In this part we create an attribute table programmatically (See 'Add new address and save it as (*.gpkg) file' section in annex), this table contains five attributes to store necessaire address parts (admirative address, a neighborhoods name, door number, apartment number), the table also stores the full name as a string of character and every new input is assigned automatically to an ID. 


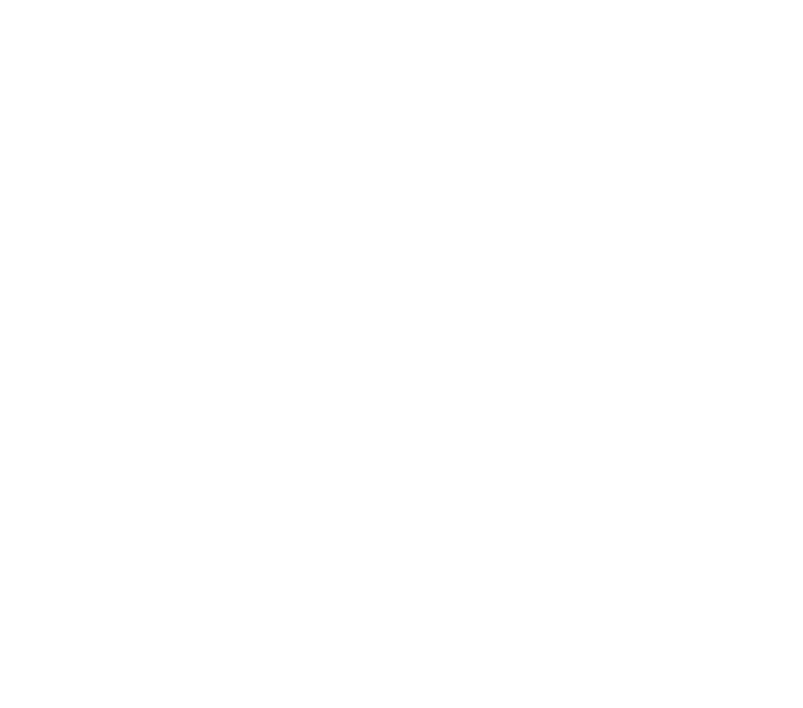

Figure 5. Add new address from field

The next figure (Figure 5) shows the result of executing the previous command.

\section{Show information about selected item}

The search process code (See 'Show information about selected item' section in the annex) is dynamic so we can search by name or by address and the result will show: the corresponding full name, the complete address (the door number, parcel number, street name and neighborhood ), geographic location, and any other information as needed (health status, date of recovery, last medical examination...etc.), this part of the code will facilitate patient access for medical intervention, increase healthcare quality as a result of reducing

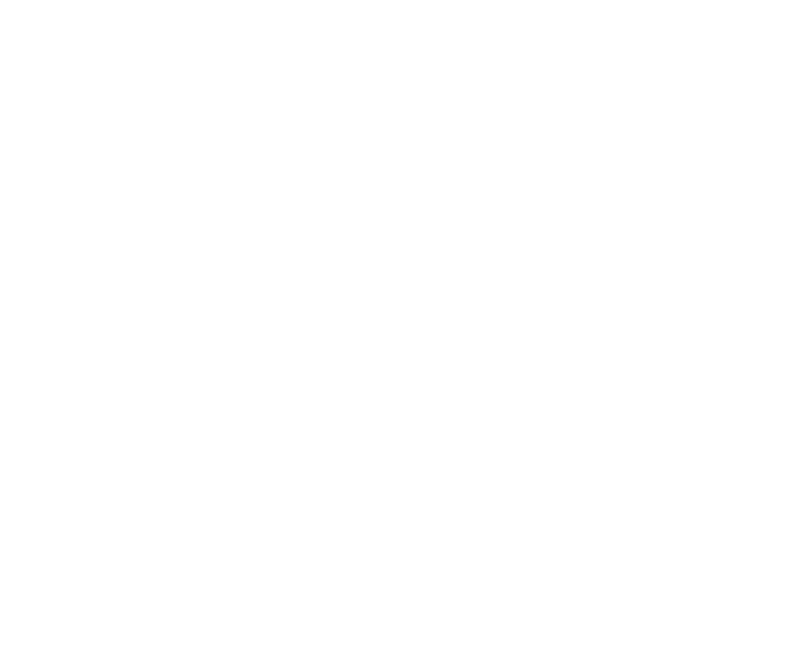

Figure 6 . Find the patient by address and display information as needed

pressure on the hospital, and help in epidemiological development analysis.

\section{Archiving and real-time information}

The main purpose of this tool is to standardize addresses and in the same time help the medical stuff to work in the field using a mobile device to collect data, especially in cases like the COVID-19 pandemic, where the ability of collected data from the field and make it available to display, to make analyses...etc., is very essential for the decision-maker. This tool is hosted by a local cartographic server that created for this purpose. Also, collecting data operation is synchronized with decision-maker who controls all operations from the control center.

\section{Conclusion}

The geocoding tool is used for crisis management and detect proximal service for healthcare which is very important in the Algerian case (Lahmar et al., 2020). In this paper we try to expand the use of geocoding as an important technic in health geography, to simplify the following data through one common platform that simplifies the exchange of information and search for it in the realtime. As the main result of this paper is to use the geocoding to handle the quarantine crisis using home healthcare. Geocoding was also used to monitor the pandemic situation in Ain Touta city and help in assembling and management of the local health resources.

To achieve the goals of the research, we based on a digital approach by accomplished a geographic database that was localized in a cartographic server and exploiting by developing an interface (application) using the $\mathrm{C \#}$ programming language to access the database in the cartographic server.
Downloading the application using smartphones and tablets which help the medical staff to work directly on the ground and make it easy to do statistics like census and update data periodically, so that every intervention in the field is recorded and stored the process on the server at the level of the crisis management center.

Among most important results are:

- Contribute significantly to the success of the quarantine and social isolation.

- Using the home healthcare improved the health system flexibility and extended its capacity from capacity 40 patients in the hospital to over $200 \mathrm{pa}$ tients using home healthcare.

- Using objective inquiry to determine the needs of the patients.

- Determine the type of disease and medication for those with chronic diseases to benefit from periodic home healthcare. 
- Using the spatial statistics to know the number and distribution of patients in each neighborhood, and to determine the geographical area of each health facility.

- Using network analysis and geocoding to determine addresses and travel paths between the health facility and patient's homes.

- Increase principle of the proximity in services so every facility assures the need of the nearest population, as a result the territory will be more hierarchy.
This paper is treated 4 main issues at the same time: Crisis management, database, and data flow management in the territory, and simplify the territory problems; in our case, we handle the unstructured addresses, using a server to store, display, and analyses data in order to generalize this approach throughout any territory, it is necessary to fill and preserve information and ensure that data input in real-time.

\section{References}

Arctur, D., \& Zeiler, M. (2004). Designing Geodatabases: case studies in GIS data modeling. ESRI, Inc.

Bonner, M. R., Han, D., Nie, J., Rogerson, P., Vena, J. E., \& Freudenheim, J. L. (2003). Positional accuracy of geocoded addresses in epidemiologic research. Epidemiology, 14(4), 408-412.

Cayo, M. R., \& Talbot, T. O. (2003). Positional error in automated geocoding of residential addresses. International journal of health geographics, 2(1), 10.

Davis, C. A., \& Fonseca, F. T. (2007). Assessing the certainty of locations produced by an address geocoding system. Geoinformatica, 11(1), 103-129.

Dearwent, S. M., Jacobs, R. R., \& Halbert, J. B. (2001). Locational uncertainty in georeferencing public health datasets. Journal of Exposure Science \& Environmental Epidemiology, 11(4), 329-334.

Edwards, S. E., Strauss, B., \& Miranda, M. L. (2014). Geocoding Large Population-level Administrative Datasets at Highly Resolved Spatial Scales. Transactions in GIS, 18(4), 586-603.

Karimi, H. A., Durcik, M., \& Rasdorf, W. (2004). Evaluation of uncertainties associated with geocoding techniques. Computer-Aided Civil and Infrastructure Engineering, 19(3), 170-185.

Krieger, N., Chen, J. T., Waterman, P. D., Soobader, M.J., Subramanian, S., \& Carson, R. (2002). Geocoding and monitoring of US socioeconomic inequalities in mortality and cancer incidence: does the choice of area-based measure and geographic level matter? the Public Health Disparities Geocoding Project. American journal of epidemiology, 156(5), 471-482.

La Direction Technique Chargée des Statistiques Régionales, l'Agriculture et de la C. (2008). Collections Statistiques $n^{\circ}$ 163/2011 Série S: Statistiques Sociales Armature urbaine - RGPH 2008 -. Office National des Statistiques. http://www.ons.dz/IMG/ pdf/armature urbaine 2008.pdf

Lahmar, B., Dridi, H., \& Akakba, A. (2020). Territorial health approach outputs of geo-governance of health facilities: case study of Batna, Algeria. Geo-
Journal, 1-15. https://doi.org/10.1007/s10708-02010189-1

Longley, P. A., Goodchild, M. F., Maguire, D. J., \& Rhind, D. W. (2005). Geographic information systems and science. John Wiley \& Sons.

Mazumdar, S., Rushton, G., Smith, B. J., Zimmerman, D. L., \& Donham, K. J. (2008). Geocoding accuracy and the recovery of relationships between environmental exposures and health. International Journal of Health Geographics, 7(1), 13.

Oliver, M. N., Matthews, K. A., Siadaty, M., Hauck, F. R., \& Pickle, L. W. (2005). Geographic bias related to geocoding in epidemiologic studies. International Journal of Health Geographics, 4(1), 29.

Präger, M., Kurz, C., Böhm, J., Laxy, M., \& Maier, W. (2019). Using data from online geocoding services for the assessment of environmental obesogenic factors: a feasibility study. International journal of health geographics, 18(1), 13.

Ratcliffe, J. H. (2001). On the accuracy of TIGER-type geocoded address data in relation to cadastral and census areal units. International Journal of Geographical Information Science, 15(5), 473-485.

Ratcliffe, J. H. (2004). Geocoding crime and a first estimate of a minimum acceptable hit rate. International Journal of Geographical Information Science, 18(1), 61-72.

Roongpiboonsopit, D., \& Karimi, H. A. (2010). Comparative evaluation and analysis of online geocoding services. International Journal of Geographical Information Science, 24(7), 1081-1100.

Ward, M. H., Nuckols, J. R., Giglierano, J., Bonner, M. R., Wolter, C., Airola, M., et al. (2005). Positional accuracy of two methods of geocoding. Epidemiology, 16(4), 542-547.

Whitsel, E. A., Quibrera, P. M., Smith, R. L., Catellier, D. J., Liao, D., Henley, A. C., \& Heiss, G. (2006). Accuracy of commercial geocoding: assessment and implications. Epidemiologic Perspectives \& Innovations, 3(1), 8 . 
Whitsel, E. A., Rose, K. M., Wood, J. L., Henley, A. C., Liao, D., \& Heiss, G. (2004). Accuracy and repeatability of commercial geocoding. American journal of epidemiology, 160(10), 1023-1029.

Yang, D.-H., Bilaver, L. M., Hayes, O., \& Goerge, R. (2004). Improving geocoding practices: evaluation of geocoding tools. Journal of medical systems, 28(4), 361-370.
Zandbergen, P. A. (2007). Influence of geocoding quality on environmental exposure assessment of children living near high traffic roads. BMC $p u b$ lic health, 7(1), 37.

Zandbergen, P. A. (2014). Ensuring confidentiality of geocoded health data: Assessing geographic masking strategies for individual-level data. Advances in medicine, 2014.

\section{ANNEX}

\section{Initiate database and necessary files}

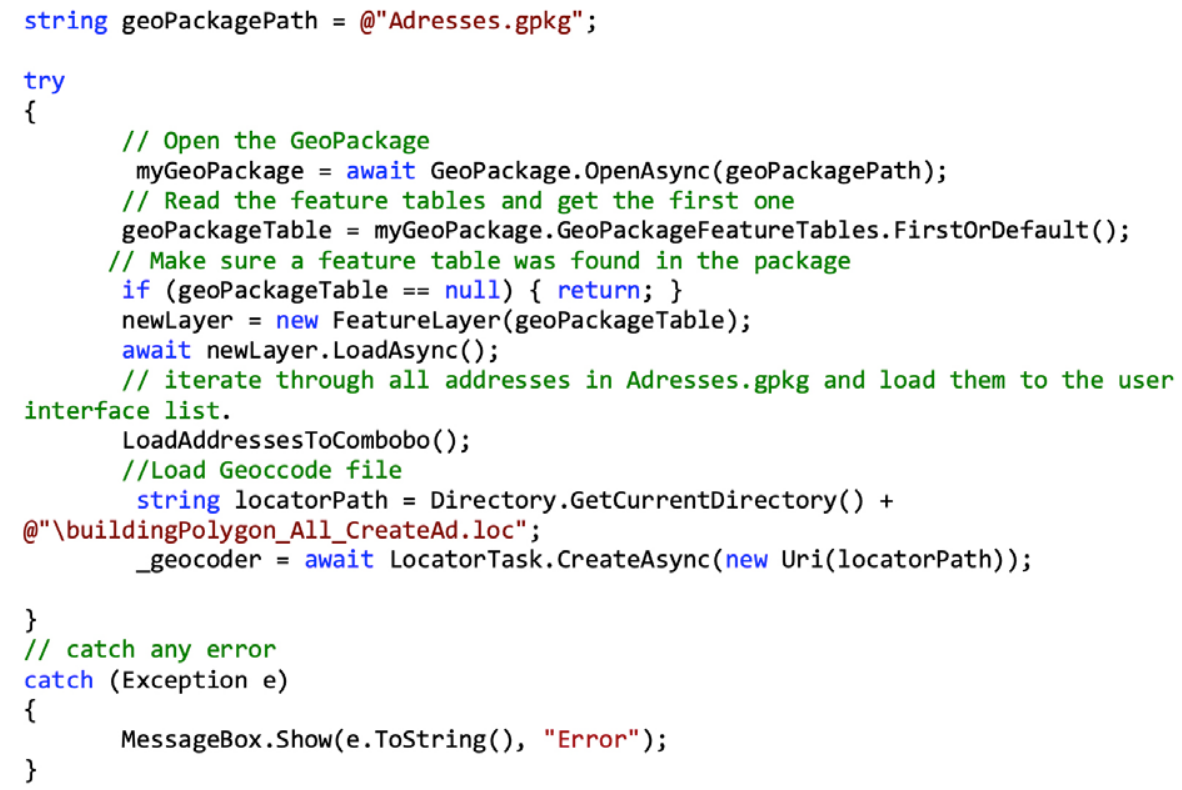

Add new address and save it as (*.gpkg) file

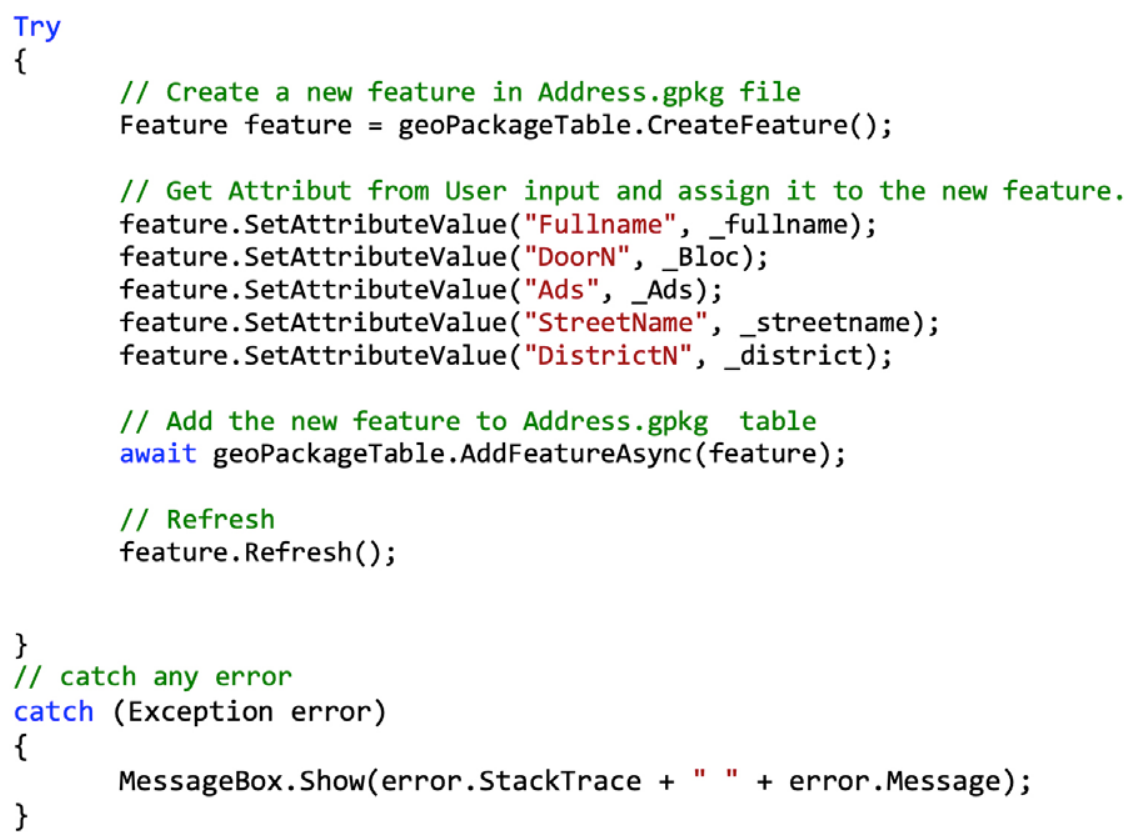




\section{Show information about selected item}

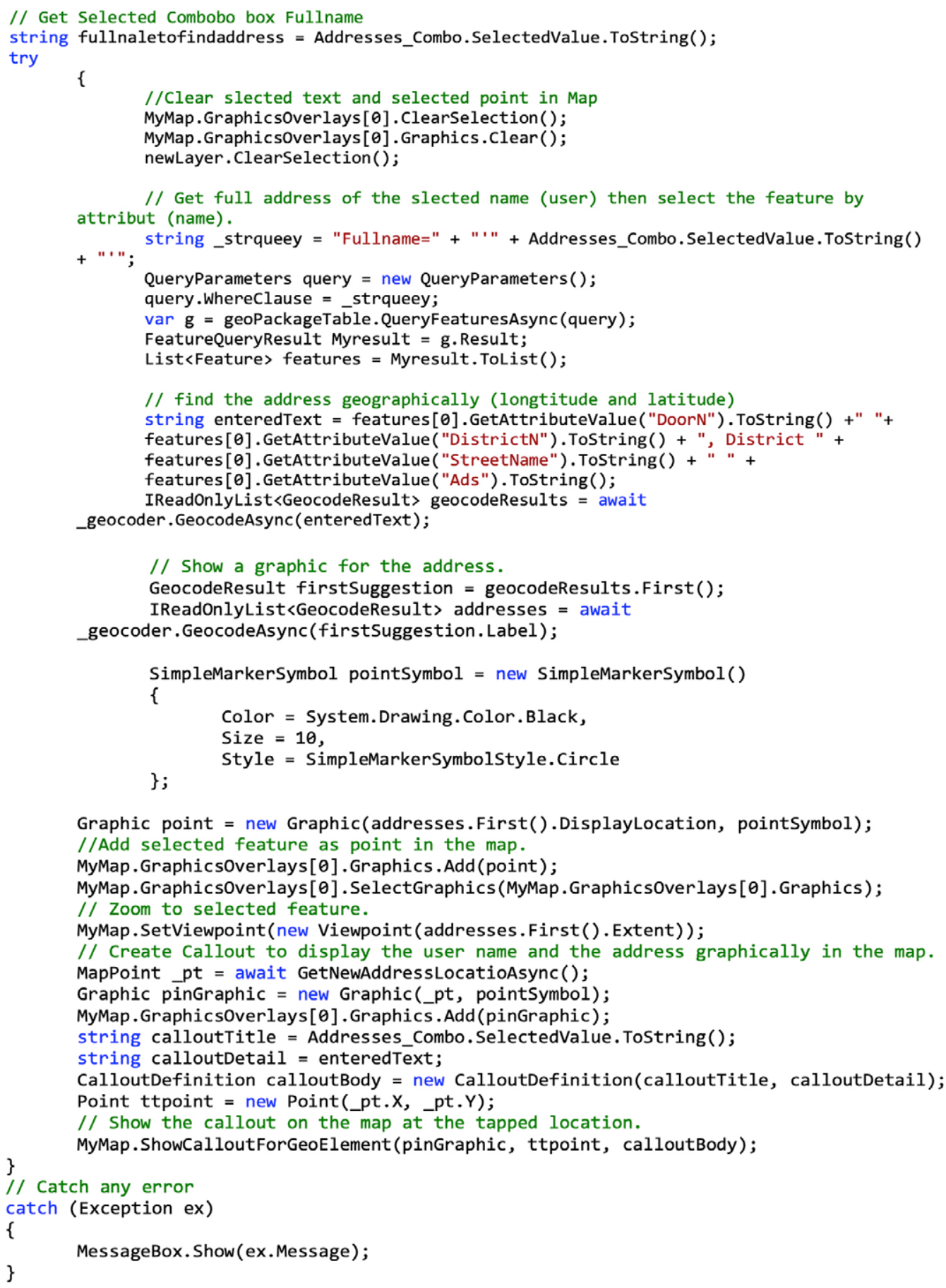

